Bull. Austral. Math. Soc.

VOL. 37 (1988) [477-478]

\title{
ORBITS AND CHAOS IN DYNAMICAL SYSTEMS
}

\section{Paul Cleary}

Chaos is a widespread phenomenon occuring in dynamical systems in such diverse fields as fluid dynamics, astrophysics, electronics, plasma physics and biology. It is the aim of this thesis to study the global properties of systems, such as their integrability, and their local properties, such as the topology of their orbit families, for several different dynamical problems.

An $n$-dimensional system is integrable if it possesses $n$ isolating integrals of the motion. Integrable systems are the exception and not the rule. One tool currently being used to examine their integrability is Painleve Analysis. This is an analytic techuique based on singularity analysis in the complex time plane. In this thesis the effects of generalising the analysis by enlarging the class of admissible singuarities we have examined, and a selection of two dimensional quartic polynomial potentials which were either integrable or close to integrable, found. The integrability of some of these potentials was confirmed by explicit analytic calculation of the second integrals of the motion and numerical Surface of Section calculations.

A classification scheme for families of periodic and quasi-periodic orbits based on both the geometry of the orbits and their topological structure in the Surface of Section was developed. This classification scheme was then used to describe and classify all the orbits occuring in the previously located quartic potentials.

There appears to be a strong relationship between the periodic orbits existing in a potential and its integrability. The KAM theorem gives sufficient conditions on the existence of invariant curves in the Surfaces of Section of non-integrable potentials. There is no converse theorem about which invariant curves definitely do break up under perturbation. However it was found that some rational invariant curves definitely do exist in non-integrable potentials. This leads to the idea of a potential being locally integrable about a periodic orbit if all the rational invariant curves, (corresponding to tori in phase space with rational winding numbers), surrounding a periodic orbit in the Surface of Section exist. There is also a strong relationship between the topology of families of regular orbits, (a local property), and the integrability of the potential,

Received 18 December 1988. Thesis submitted to Monash University June 1987. Degree approved November 1987. Supervisor: Dr. J.J. Monaghan.

Copyright Clearance Centre, Inc. Serial-fee code: 0004-9729/88 \$A2.00+0.00. 
(a global property). In particular a chaotic orbit exists between any two families of quasi-periodic orbits which can not be smoothly deformed from one to the other. Also perturbing integrable potentials, causes topological changes in the corresponding Surface of Section in very precise ways. This behaviour is exhibited by all the integrable quartic potentials. The changes are intimately related to the set of lowest resonance unstable periodic orbits.

Several methods for calculating Lyapunov exponents for dynamical systems have been developed. Three of these were compared for a variety of non-conservative systems. The best one was then used to calculate the spectrum of the largest chaotic orbit in each of the previously mentioned quartic polynomial potentials. A direct correlation was found to exist between the magnitude of the maximal Lyapunov exponent and the area of the corresponding chaotic region in the Surface of Section.

The triaxial Schwarzschild potential is used in galactic dynamics to model elliptical galaxies and some special features such as dust lanes. We examined the periodic orbit structure of the rotating Schwarzschild potential and found seven new families in addition to the nine previously known ones. The periodic orbit bifurcations and corresponding changes in stability were found to obey very special rules. The types of bifurcations occuring are all of the flip bifurcation and the saddle-node bifurcation types arising in one dimensional mappings. The bifurcation structures are of two types: vertically or horizontally resonant instability strips in the equatorial plane orbits and complex three dimensional cascades.

Department of Mathematics

Monash University

Clayton, Victoria, 3168

Australia 